\title{
The various patterns of dislocation injuries at the carpometacarpal joints: A case series and brief review of literature with regards to the main topics
}

\author{
Ingo Schmidt \\ Medical Centre Wutha-Farnroda, Germany
}

\begin{abstract}
Abbreviations: $\mathrm{CMCJ}(\mathrm{s})$ : carpometacarpal joint(s); OA: osteoarthritis; PA: posterior-anterior; CRPP: closed reduction and percutaneous pinning; CT: computed tomography; ORP: open reduction and pinning; MRI: magnetic resonance imaging; APLT: abductor pollicis longus tendon; ORLRP: open reduction with ligament reconstruction and pinning
\end{abstract}

\section{Introduction}

\section{Carpometacarpal Joints II-V}

Dislocation injuries of the carpometacarpal joints (CMCJs) II-V are rare accounting less than $1 \%$ only of all hand injuries, and first observations were described by Cooper and Roux in the 19th century $[1,2]$. The CMCJs II-V are saddle joints and stabilized by the weaker volar and the stronger dorsal ligaments, transverse metacarpal ligaments, the extrinsic flexor and extensor tendons, and the intrinsic muscles of the hand. The feature is that the stability of these joints decreases from radial- to ulnarwards. The CMCJs II and III in which the dynamic compressive stability is enhanced by the insertion of the flexor carpi radialis and extensor carpi radialis longus/brevis at the bases of the 2nd and 3rd metacarpal bone allow a movement of 1 to $3^{\circ}$ only whereas the CMCJ V 15 to $20^{\circ}$ respectively, and the CMCJ III in which the metacarpal articulates with the capitate bone is the strongest joint that is to be considered as the "key stone" due to its more proximal location than the other joints and its initial injury seems to be a predisposition of dislocation injuries at the other CMCJs [36]. Thus, the frequency of dislocation injuries increases from radial- to ulnarwards. Of all solitary injuries the CMCJ V is involved in $50 \%$ of cases (Case Presentation 1), and in the more common multiple injuries the dislocations involve both in $30 \%$ the CMCJs II -V and the CMCJs IV-V that can or cannot to be associated with additional fractures of the metacarpal bases (Case Presentation 2) [7-9]. Note that coexistent fractures at the distal carpal row are observed in up to $26 \%$ of cases, and isolated dislocation injuries at the CMCJ V can be accompanied in up to $97 \%$ with fractures of the 5 th metacarpal base mostly observed if dislocation occurs in dorsal direction [10,11].

Dislocation injuries of the CMCJs II-V most commonly resulting from high-energy trauma wrist trauma. Direction of dislocation is mostly observed in dorsal direction and occurs by axial loading of the metacarpal bones when the CMCJs are held in flexion, whereas a direct blow to the lateral aspect of the 5th metacarpal base can produce a dislocation at the CMCJ V in volar direction. Simultaneous dislocation injuries of the CMCJs II-V in volar direction, first reported by Tricksen in 1872, are very uncommon and can be initially associated with an acute compression syndrome of the median and/or ulnar nerve. Depending on the integrity of the flexor carpi ulnaris tendon and/or the pisometacarpal ligament, dislocation injuries of the CMCJ V can be further subclassified as radial or ulnar [3,4,12-18].

One of the major problem is that these injuries can be easily overlooked in the emergency setting if both clinicians and radiologists do not apply a high index of suspicion which can potentially lead to early neurovascular impairment (i.e. compartment syndrome). Furthermore, if primarily undiagnosed and untreated or recurrence occurs especially in cases of initial subluxations (i.e. dynamic instability) only, the injury is followed by chronic instability-related residual pain, poor functional outcome, complex regional pain syndrome, and/or post-traumatic osteoarthritis (OA). For those cases, the fusion of the unstable CMCJs remains inevitable secondarily (Case Presentation 3). Initial or postoperative posterior-anterior (PA) radiographs after closed reduction and percutaneous pinning (CRPP) do not always show the typical bony overlapping at the CMCJs (i.e. elapsed Gilula's "M-line"). Hence, for initial and early (!) postoperative assessment of joint alignment and/or concomitant metacarpal base fracture, oblique and lateral radiographs and/or computed tomography (CT) especially with the sagittal plane are absolutely required (Case Presentation 2). Primary open reduction and pinning (ORP) is inevitable if neurovascular impairment is present, and secondarily after failed CRPP mainly based on soft tissue interposition (Case Presentations 1 and 2). Primary conservative treatment (i.e. closed reduction without pinning) can be an option if stability of joints can be safely assessed [3-18].

\section{Case Presentation 1}

A 57-year-old female sustained a left ligamentous dislocation injury at her CMCJ V in dorsoulnar direction after a fall from $1,5 \mathrm{~m}$ height (Figure 1A). On clinical examination, there was painful swelling associated with an internal rotation deformity of her 5th finger. CRPP was initially performed but it was unsuccessful; thus, ORP became necessary. Intraoperatively, soft tissue interposition into the CMCJ V was found as cause for failed CRPP. Surgical stabilization of the CMCJ

${ }^{*}$ Correspondence to: Ingo Schmidt, Medical Centre Wutha-Farnroda of the Hospital Bad Salzungen (Teaching Hospital of the Friedrich-SchillerUniversity Jena, Germany), Gothaer Str. 67, 99848 Wutha-Farnroda, Germany / Lindigallee 3, 36433 Bad Salzungen, Germany, Tel: 00491789503035; E-mail: schmidtingo62@googlemail.com

Key words: Carpometacarpal joints, Dislocation injuries

Received: July 15, 2018; Accepted: July 23, 2018; Published: July 31, 2018 
$\mathrm{V}$ was done by $2 \mathrm{~K}$-wires with an additional tension wire after removal of intra-articular soft tissue fragments and its anatomical reduction (Figure 1B). The left wrist and hand was immobilized by a plaster splint for 6 weeks, after that, all wires were removed, and strengthening was started. Ten weeks after surgery, the patient could be fully recovered in the absence of pain and without functional deficits.

\section{Case Presentation 2}

A 24-year-old male sustained a polytrauma after a high-energy car accident with multiple fractures of both lower extremities, a left complete forearm shaft fracture, a left metacarpal V shaft fracture, a left clavicle fracture, and multiple rib fractures with a right hematothorax. Additionally, there was a massive swelling on the dorsal aspect of his overall right hand without any signs of neurovascular impairment, and PA radiograph revealed complete dislocations at the CMCJs II-V in dorsal direction (Figure 2A). Firstly, a CLPP was done and intraoperative fluoroscopy revealed falsely an anatomical reduction of all injured joints with a correct Gilula's "M-line" (Figure 2B). For postoperative assessment of joint congruencies, a CT of the right hand was performed 2 days after surgery that revealed in the coronal plane (analogue to the intraoperative fluoroscopy) a correct Gilula's "M-line", but in the sagittal plane persistent subluxations at all CMCJs II-V associated with an increase of failed joint alignment from radialto ulnarwards, and additionally, metacarpal base fractures II / IV / V
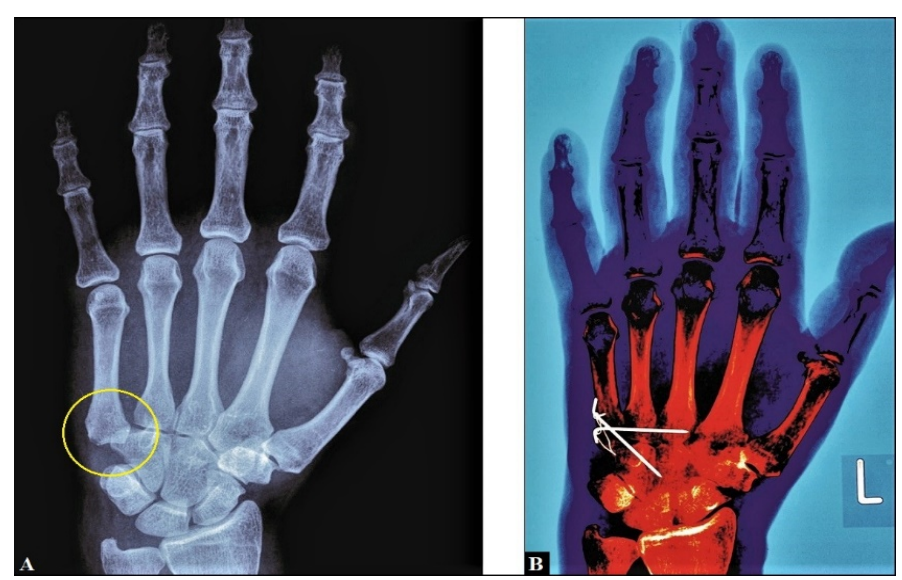

Figure 1 (Case Presentation 1): (A) Initial PA radiograph showing CMCJ V dislocation in dorsoulnar direction with bony overlapping between the 5th metacarpal and hamate bone related to an elapsed Gilula's "M-line" (yellow arrow). (B) PA radiograph after ORP showing correct alignment at the CMCJ V
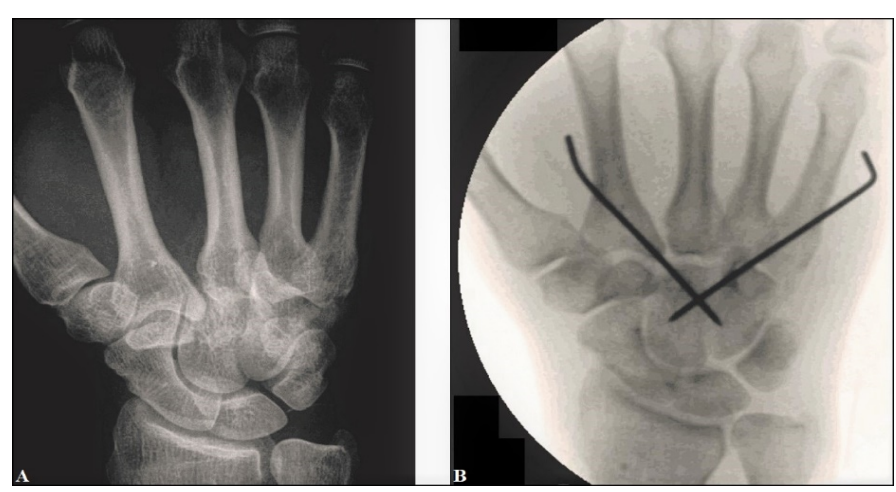

Figure 2 (Case Presentation 2): (A) Initial PA radiograph showing dislocations at the CMCJs II-V in dorsal direction, note the elapsed Gilula's "M-line". (B) Intraoperative fluoroscopy after CRPP demonstrating falsely a correct Gilula's "M-line" which pretends a proper alignment at the CMCJs II-V at its dorsal aspects (Figures 3A-D). Secondarily, an ORP had to be performed 3 days after initial surgery (Figure $4 \mathrm{~A}$ ). The right wrist and hand was immobilized by a plaster splint for 6 weeks, after that, all $\mathrm{K}$-wires were removed, and strengthening was started. PA and oblique radiographs revealed at this time correct CMCJ II-V alignments (Figure $4 \mathrm{~B})$. One year after injury, an excellent right wrist and hand function was evident in the absence of pain (Figure 5).

\section{Case Presentation 3}

A 26-year-old male reported at his first presentation in our hospital about disability and pain around his right wrist accompanied with persistent loss of grip strength of his right hand after a fall from $2 \mathrm{~m}$ height 1 year previously that was initially treated with the diagnosis "wrist sprain" by immobilization using a soft orthosis for 4 weeks in another institution. On clinical examination, instabilities at the CMCJs IV and V could be found with a palpable and audible clicking under the condition of manual pressure onto the dorsal aspect of the metacarpal IV and $\mathrm{V}$ bases as result of closed reduction at these joints when subluxations in dorsal direction were provoked before by maximal passive flexion of the wrist and metacarpophalangeal joints II-V by the examiner. Magnetic resonance imaging (MRI) revealed persistent incomplete disruptions of the capsule-ligamentous complexes at these joints, thus, definitive fusions of the CMCJs IV and V were detected by us. The CMCJs IV and V were exposed through a dorsal incision with careful preservation of the dorsal branch of ulnar nerve (Figure 6A). MRI diagnosis with disruptions of the capsule-ligamentous complexes at these joints could be confirmed intraoperatively (Figure 6B). The corresponding cartilage joint surfaces were resected, and then 2 locking 2,4 mm titanium plates were fixed proximally each at the hamate bone, and distally at metacarpals IV and V creating a V-configuration (Figure 6C). After that, joint cavities were filled off with cancellous bone chips which were taken out of the the right distal radial metaphysis. Radiographically, there was a correct postoperative alignment in both joints, and correct positioning of both fusion plates (Figure 6D). Postoperatively, the right wrist and hand was immobilized in a plaster splint for 3 weeks, then, motions were freed, and strengthening was started 6 weeks after surgery. Ten weeks after surgery, the patient could be recovered fully in his original occupation as a mechanic with a satisfactory pain relief. One year after surgery, complete bony unions of both joints could be evaluated, but he reported intermittent disability due to tenosynovitis caused by mechanical irritation of the extensor tendons by the fusion plates; hence, both plates had to be removed (Figure 7A). Six weeks later, the patient was satisfied again in the presence of good functional outcome (Figure 7B).

\section{Carpometacarpal joint I (thumb)}

Biomechanically, the CMCJ I can be best described as a "twisted saddle" with 2 axes for extension-flexion and abduction-adduction only, and there are several volar and especially the major strong dorsal ligaments which provide joint stability [19]. The mean range of motion in CMCJ I of healthy subjects is $27,3^{\circ}$ to $41^{\circ}$ for extension-flexion, $51^{\circ}$ to $66,9^{\circ}$ for abduction-adduction, and $10^{\circ}$ to $21^{\circ}$ for axial rotation, associated with significant differences between females and males, but no differences between right and left hands; and the maximum estimated contact area on the trapez and 1st metacarpal bones is in palmar abduction whereas the minimum is in adduction [20,21]. Key pinch as well as object grasp demonstrate a coupled motion: for key pinch the 1st metacarpal bone undergoes volar translation-internal rotation-flexion, for object grasp the 1st metacarpal bone undergoes ulnar translation-flexion-abduction [22]. Thumb's circumduction, 

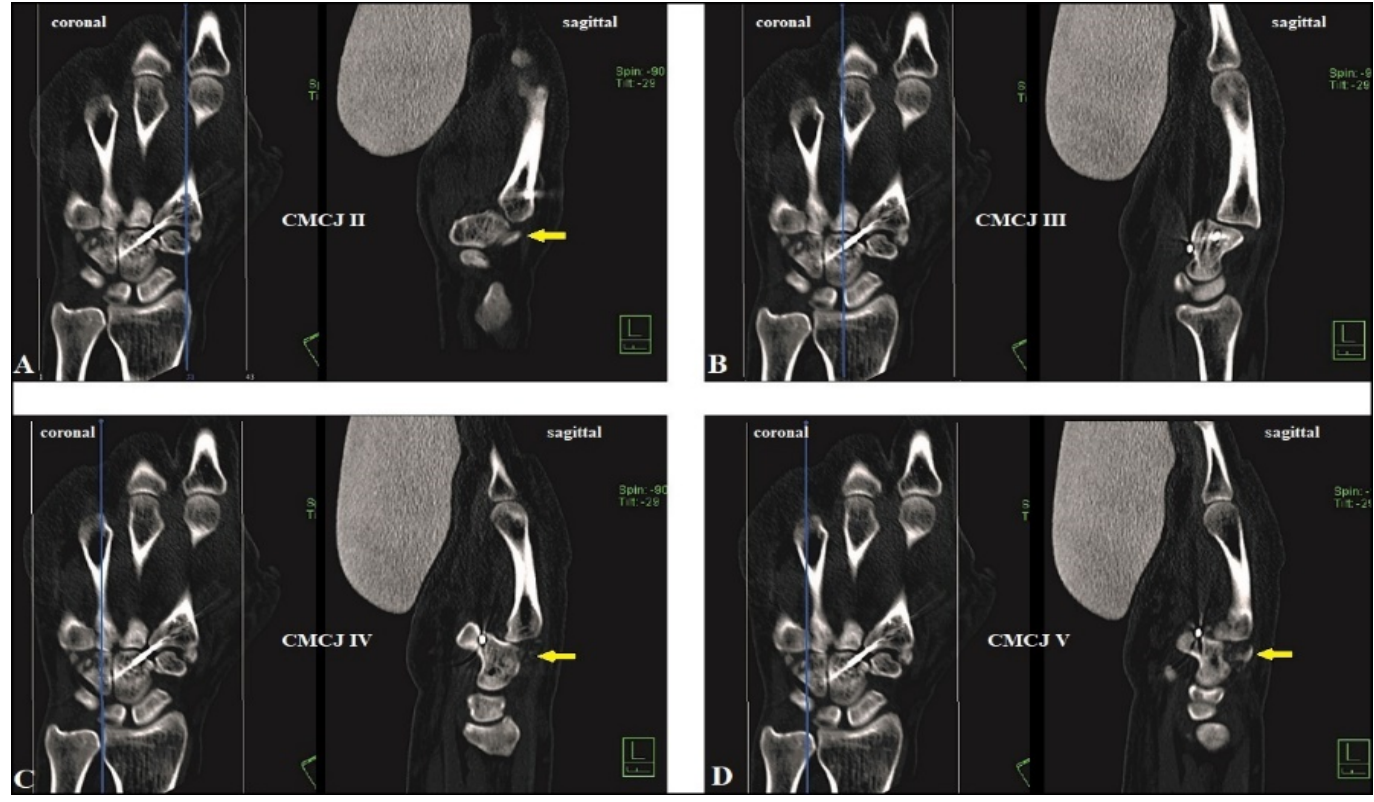

Figure 3 (Case Presentation 2, CT scans 2 days after CRPP, note that in all coronal planes an anatomically reduction at all CMCJs II-V could be suggested, and the severity of persistent subluxations showing an increase from radial- to ulnarwards in the sagittal planes): (A) Sagittal CT scan of the CMCJ II showing unchanged subluxation in dorsal direction, note the additional metacarpal II base fracture at the dorsal aspect (yellow arrow). (B) Same situation at the CMCJ III as in CMCJ II but without an additional metacarpal III base fracture. (C) Nearly complete luxation at the CMCJ IV in dorsal direction, note the multiple little bony fragments out of the metacarpal IV base at the dorsal aspect (yellow arrow). (D) Same situation in $\mathrm{CMCJ} \mathrm{V}$ as in $\mathrm{CMCJ} I V$, note the additional metacarpal $\mathrm{V}$ base fracture at dorsal aspect (yellow arrow)
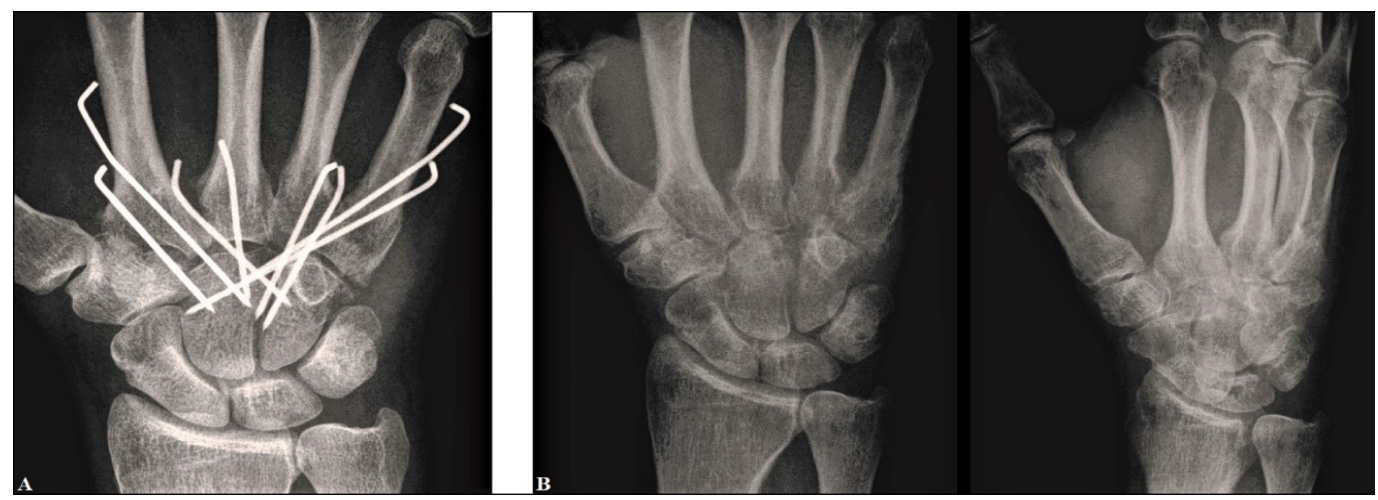

Figure 4 (Case Presentation 2): (A) PA radiograph after secondarily performed ORP. (B) PA and oblique radiographs after removal of all K-wires, there is a correct alignment at all CMCJs II-V

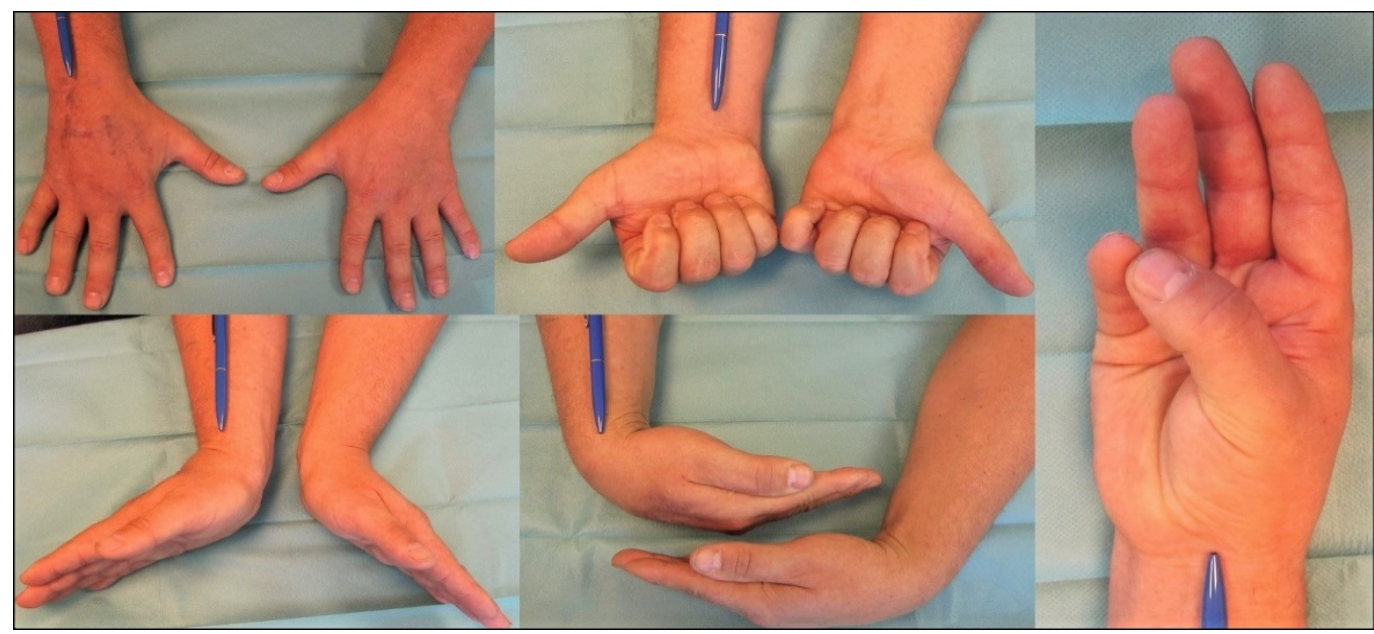

Figure 5 (Case Presentation 2): Clinical photographs demonstrating excellent functional recovery at the 1-year follow-up 

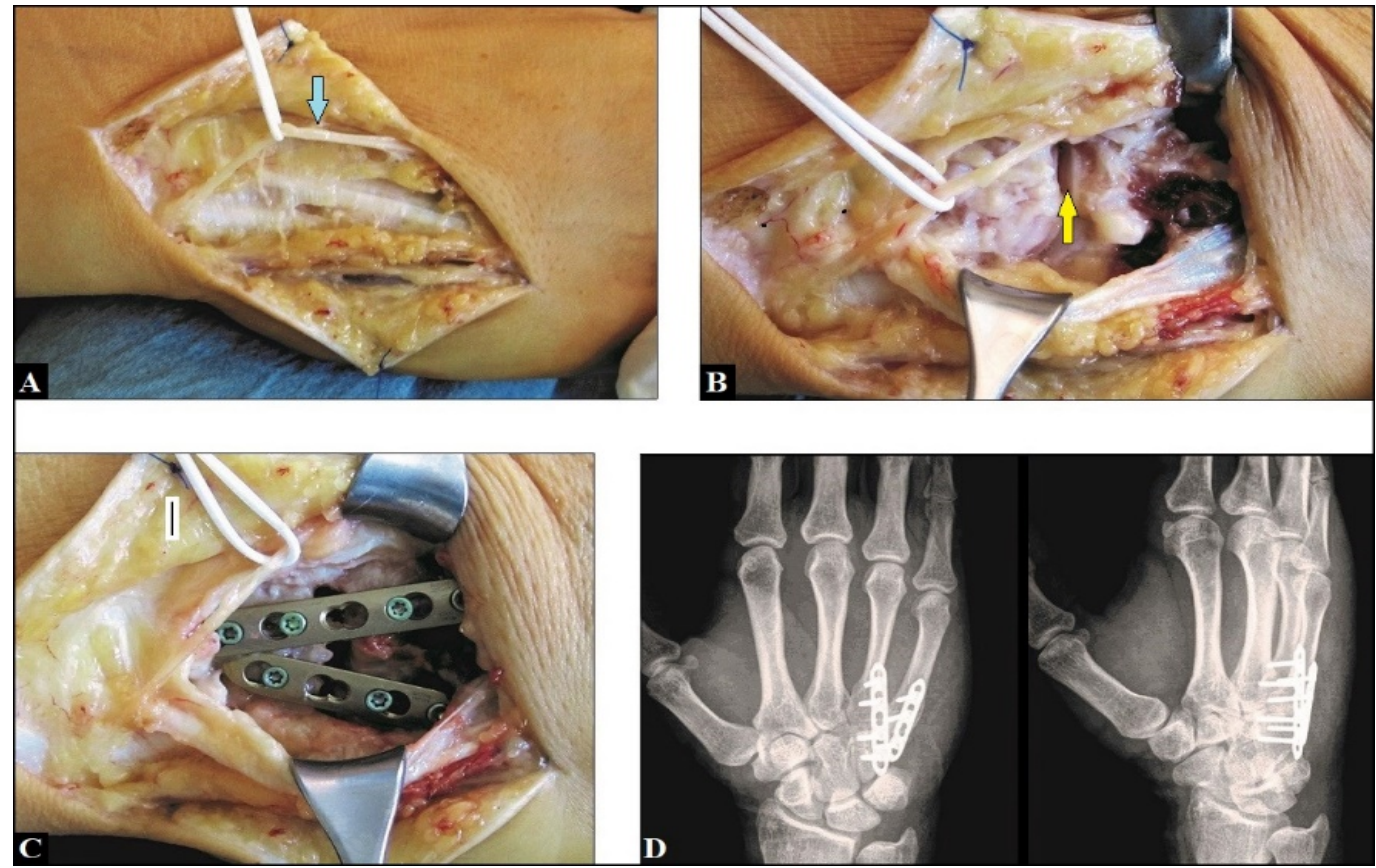

Figure 6 (Case Presentation 3): (A) Intraoperative clinical photograph showing surgical approach through the dorsal incision with careful preservation of the dorsal branch of ulnar nerve (light blue arrow). (B) Intraoperative clinical photograph showing exposure of the CMCJs IV and V with disruptions of its capsule-ligamentous complexes (yellow arrow). (C) Intraoperative clinical photograph showing positioning of both fusion plates creating a V-configuration. (D) Postoperative PA and oblique radiographs demonstrating correct alignments at the CMCJs IV and $\mathrm{V}$, and correct positioning of both fusion plates
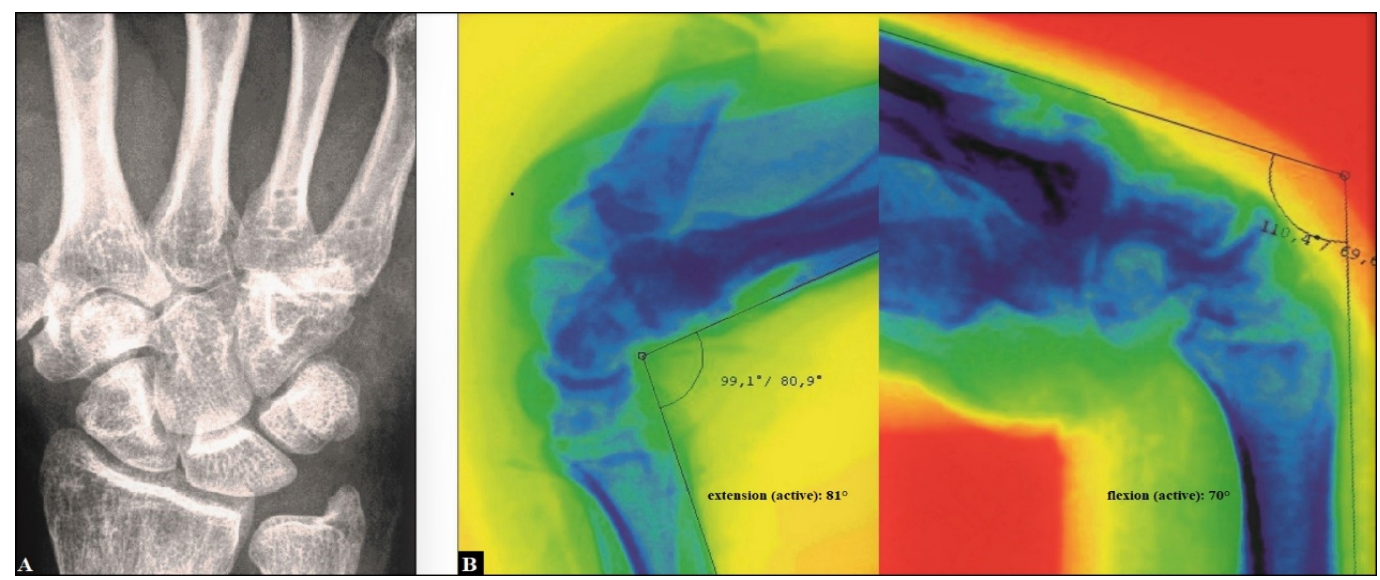

Figure 7 (Case Presentation 3): (A) PA radiograph demonstrating removal of both fusion plates and uneventful bony unions of the former CMCJs IV and V joints. (B) Dynamic fluoroscopy at terminal active ranges of motion 6 weeks after plate removals demonstrating excellent wrist function

that requires a "third functioning axis" for pronatoric rotation (i.e. opposition), is a result of evolution in hominid species as a functional adaption to stand upright, freeing the torso and upper limbs without any essential changes in its anatomically determined articulation, respectively [22]. Unfortunately, the great mobility that is seen invivo in these 3 functioning axes like a "cardan joint" makes that joint intrinsically unstable and primary $\mathrm{OA}$ is "the price of an opposable thumb" [23]. However, patients undergoing repetitive microtraumata by longstanding heavy work-related loads may have an up to 12fold higher risk for development of post-traumatic CMCJ I OA $[24,25]$. Furthermore, neglected injuries or incomplete reduction of CMCJ I dislocations causes chronic instability and muscle imbalance potentially leading to painful post-traumatic OA as well $[26,27]$. For advanced stage of CMCJ I OA there are mainly 3 salvage options in treatment: total CMCJ I replacement, an excisional procedure (i.e. trapeziectomy alone or with an additional ligament reconstruction
/ ligament interposition / suspension), or CMCJ I fusion (Figures $8 \mathrm{~A}-\mathrm{C})[28,29]$.

CMCJ I dislocations are rare accounting approximately less than $1 \%$ of all hand injuries as well, and they are mostly pure ligamentous origins following axial loading of the 1st metacarpal bone when the thumb is held in flexion at its metacarpophalangeal joint. In 1954 Gedda first mentioned CMCJ I dislocation without skeletal trauma associated with loss of function of the thumb without Bennet's fracture, and in 1976 Harrey used the phrase "Bennett's fracture without a fracture" when instability persists after reduction. Dislocation is mostly observed in posterior direction, whereas dislocation in anterior direction is underrepresented. Furthermore, it is much more common observed in adults than in adolescents or children. Recent studies suggest that dislocation is mainly caused by disruption either of the dorsoradial ligament or the anterior oblique ligament followed by dislocation of 
the 1st metacarpal bone through the thin dorsal capsule whereas the other especially volar ligaments remain intact, and persistent instability or recurrence of dislocation is based on the persistent dynamic tension force of the abductor pollicis longus tendon (APLT) at its insertion at the base of the 1st metacarpal bone [30-41].

The major problem in diagnostic management is that partial ligament tears can lead to dynamic instability only, hence, this injury is often undiagnosed when using static conditions alone in diagnostic management. Furthermore, how can we differentiate "normal" or "idiopathic" dorsoradial subluxation from traumatic origins? Rust et al. found in a retrospective CT study involving 50 patients aged 18 to 62 years without CMCJ I disorders that significant increase of dorsoradial subluxation occurs after 46 years of age, and a mean dorsoradial subluxation of $21 \%$ (range $14 \%$ to $30 \%$ ) can be considered normal in this age group (Case Presentation 5) [42]. Hirschmann et al. noted that when using MRI the thumb should be held in the "uncomfortable" opposition in which the CMCJ I is congruent only, and so, this position allows probably the best investigation in differentiation of positionrelated subluxation from underlying post-traumatic or disease-related subluxations [43]. To our experience, radiographic assessment of CMCJ I instability under dynamic conditions seems probably to be the method of choice (Case Presentation 4). For treatment of CMCJ I dislocation injuries it is still controversial whether CRPP is or is not superior over open reduction with ligament reconstruction and pinning (ORLRP), but it must be noted that CRPP can be associated with persistent instability in up to $2 / 3$ of patients $[32,34,35,39,41,44$ -
47]. However, in order to avoid post-traumatic OA the creation of a stable and correct aligned CMCJ I utilizing varying surgical treatment options is without alternative.

\section{Case Presentation 4}

A 32-year-old female reported swelling, pain, instability, and impaired thumb's function at her right CMCJ I after a fall while alpine skiing. Radiographically, there was only a suspected widening of the space between the metacarpal bones I and II under static conditions. In contrast, under dynamic conditions (i.e. maximal passive thumb's abduction by the examiner) CMCJ I dislocation in dorsoradial direction could be safely assessed (Figure 9A). On clinical examination, the instability at the CMCJ I could be assessed as well with a palpable and audible clicking under the condition of manual pressure onto the dorsal aspect of the 1st metacarrpal bone as result of closed reduction at this joint when (sub)luxation in dorsal direction was provoked before by maximal passive flexion of the thumb by the examiner (Figure 9B). An ORLRP was detected by us, and the CMCJ I was exposed typically through a dorsal incision. Intraoperatively, a complete disruption of the dorsal capsule-ligamentous complex could be found (Figure 10A). For ligamentous reconstruction, a distally pedicled APLT slip was prepared (Figure 10B), and then 1 transverse bony tunnel each was drilled through the trapez bone and the base of the 1st metacarpal bone. After that, the APLT slip was passed through the bony tunnel of the trapez bone from ulnar- to radialwards (Figure 10C), and then rerouted to distal ulnarwards to the base of the 1st metacarpal bone
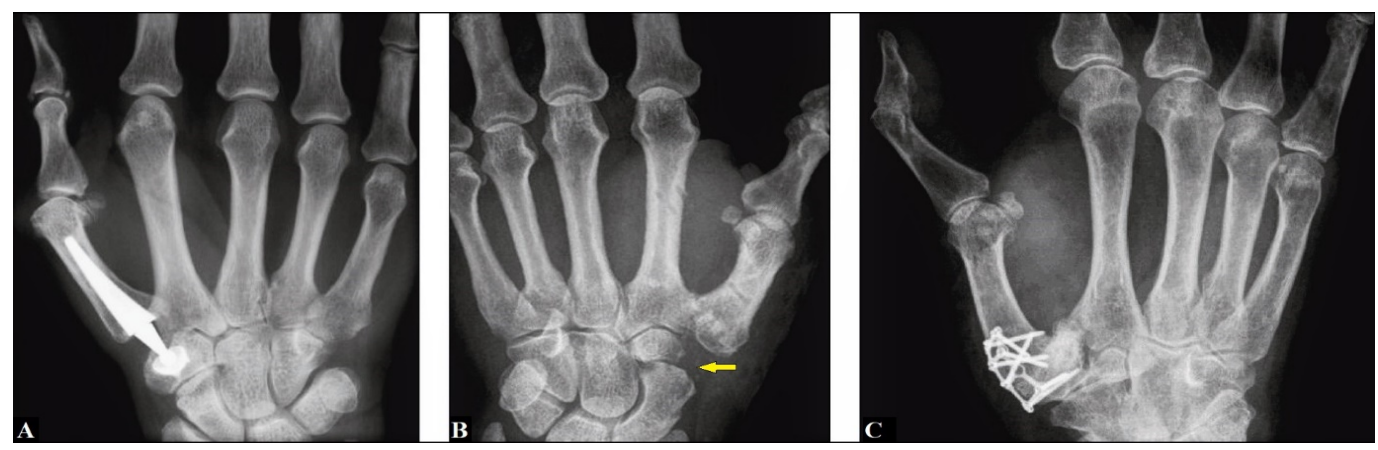

Figure 8 (Examples for salvage options in treatment of CMCJ I OA): (A) Total joint replacement. (B) Trapeziectomy alone (yellow arrow). (C) Joint fusion
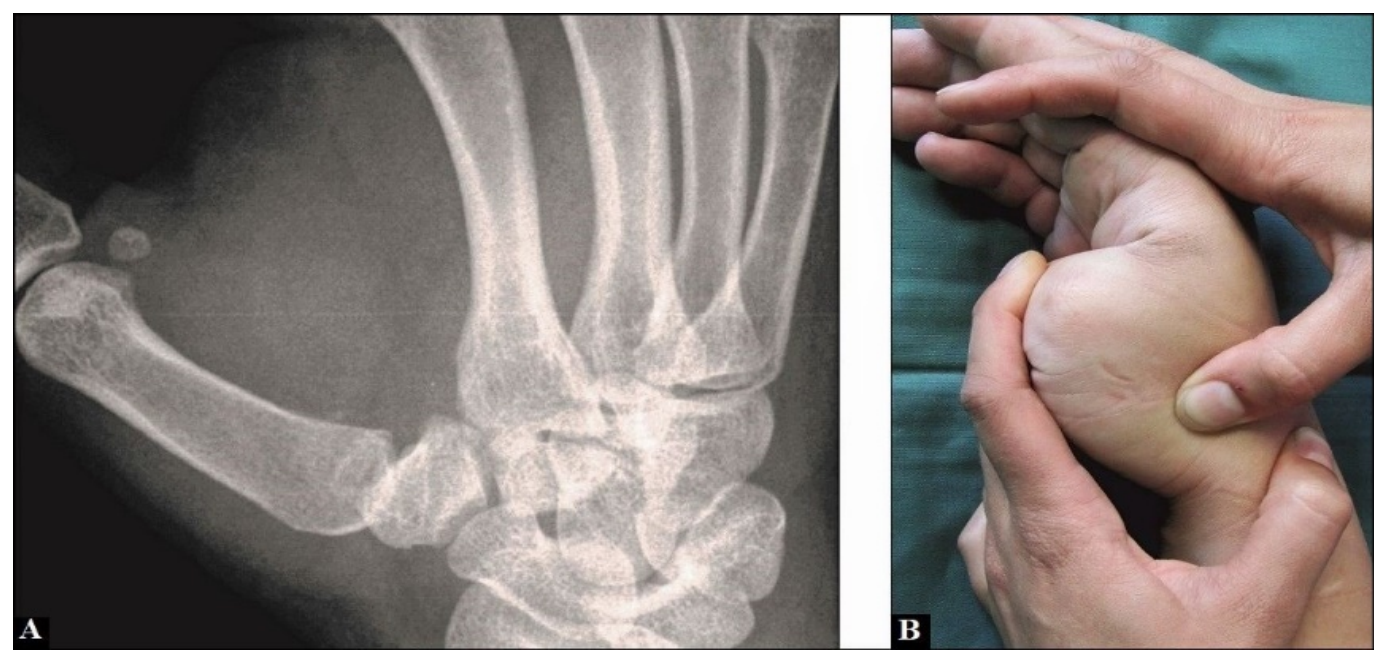

Figure 9 (Case Presentation 4): (A) Dynamic radiographic assessment of pronounced dorsoradial subluxation at the CMCJ I while maximal passive abduction by the examiner. (B) Clinical assessment of instability at the CMCJ I by the examiner 
crossing the CMCJ I and passed through the bony tunnel of the base of 1st metacarpal bone from ulnar- to radialwards again (Figure 10 D). Then, the APLT slip was rerouted to proximal ulnarwards crossing the CMCJ I again, sutured with itself at its tendinous crossing point over the CMCJ I, and the end of the tendon slip was fixed at the trapezoid bone utilizing a micro anchor (Figure 10E). At least, a metacarpal transfixation I to II using $2 \mathrm{~K}$-wires was carried out (Figure $10 \mathrm{~F}$ ). Postoperatively, the right wrist and thumb was immobilized by a plaster splint for 6 weeks, after that, all wires were removed, and strengthening was started. Ten weeks after surgery, the patient could be fully recovered in the absence of pain and without functional deficits. At the 6-months follow-up, there was unchanged a stable and correct alignment at the CMCJ I under dynamic conditions radiographically thumb's function was excellent, and the patient was very satisfied with her outcome (Figures 11A-B).

\section{Case Presentation 5}

A 36-year-old female reported swelling, pain, instability, and impaired thumb' function at her left CMCJ I after a fall while mountain bicycling. Static PA radiograph showing marked CMCJ I subluxation in radial direction (Figure 12A), and the amount of radial subluxation could be assessed exactly with more than $50 \%$ of full width of the base of the 1st metacarpal bone by CT scans (Figure 12B). MRI revealed disruption of the intermetacarpal ligament (Figure 12C). Surgical treatment and postoperative care was performed in the same techniques as described by us in Case Presentation 4, but with 2 changes: the end of the APLT slip was sutured at periost of trapezoid bone without the use of a micro anchor, and the metacarpal transfixation I to II was done with the use of $1 \mathrm{~K}$-wire only (Figure 12D). At the 6-months follow-up there was unchanged a stable and correct alignment at the CMCJ I, and the patient was satisfied with her outcome (Figures 13A-B).
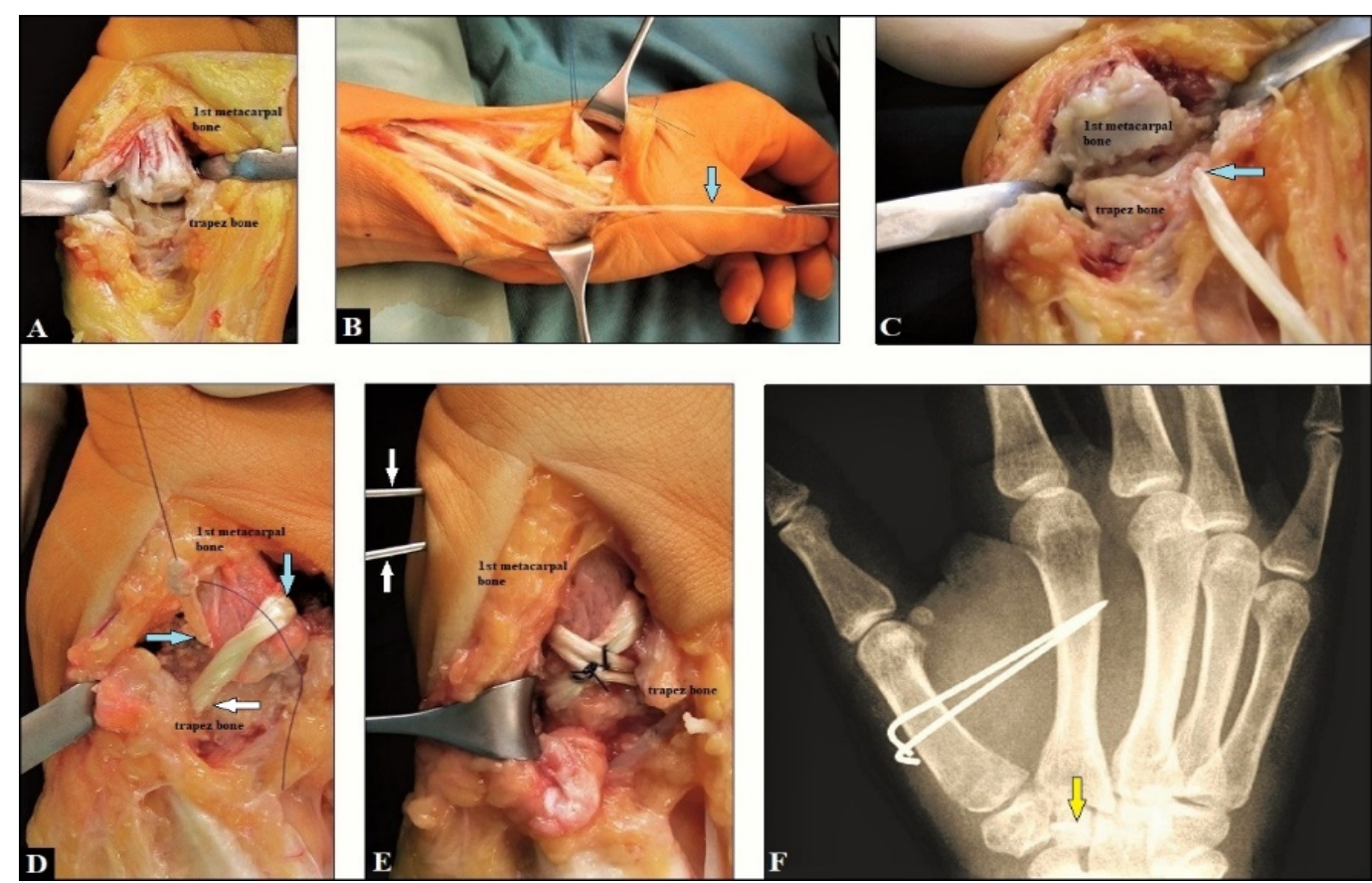

Figure 10 (Case Presentation 4): (A) Intraoperative clinical photograph showing complete disruption of the dorsal capsule-ligamentous complex. (B) Intraoperatively, preparing the distally pedicled APLT slip. (C) Intraoperatively, passing the APLT slip through the bony tunnel of trapez bone. (D) Intraoperatively, rerouting the APLT slip to distal crossing the CMCJ I and passing through the bony tunnel of the base of the 1st metacarpal bone. (E) Intraoperatively, rerouting of the APLT slip again to proximal and sutured with itself at its tendinous crossing point. The end of the APLT slip was fixed at the trapezoid bone utilizing a micro anchor. (F) Postoperative PA radiograph demonstrating the well aligned CMCJ I, the metacarpal transfixation I to II using $2 \mathrm{~K}$-wires, and the micro anchor into the trapezoid bone (yellow arrow)

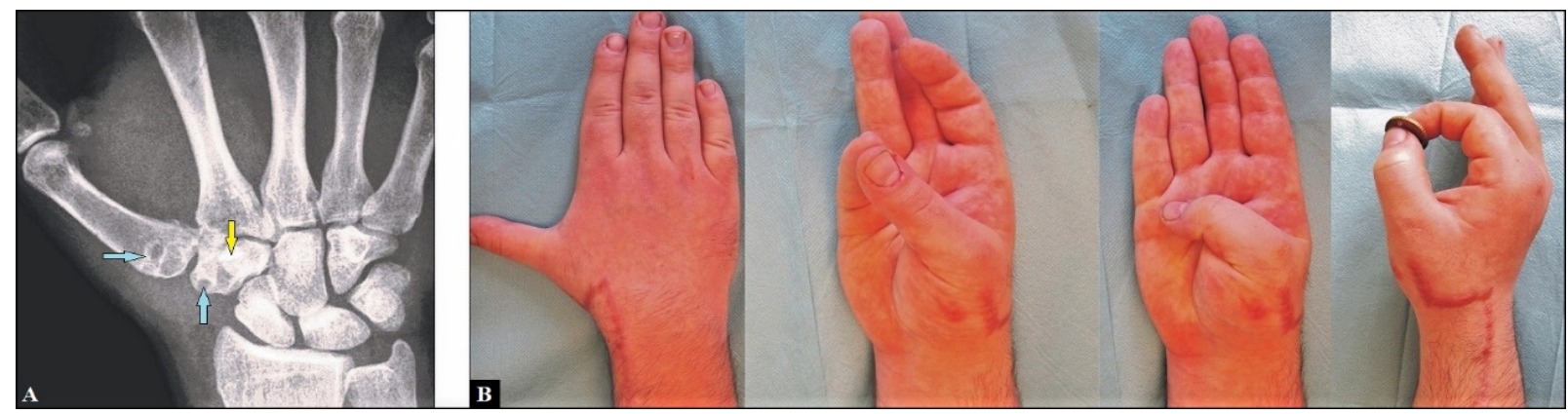

Figure 11 (Case Presentation 4, 6-months follow-up): (A) PA radiograph demonstrating unchanged correct CMCJ I alignment, note the transverse bony tunnels through the trapez bone and the base of the 1st metacarpal bone (light blue arrows) and the micro anchor into the trapezoid bone. (B) Clinical photographs showing excellent stable restoration of thumb's function 

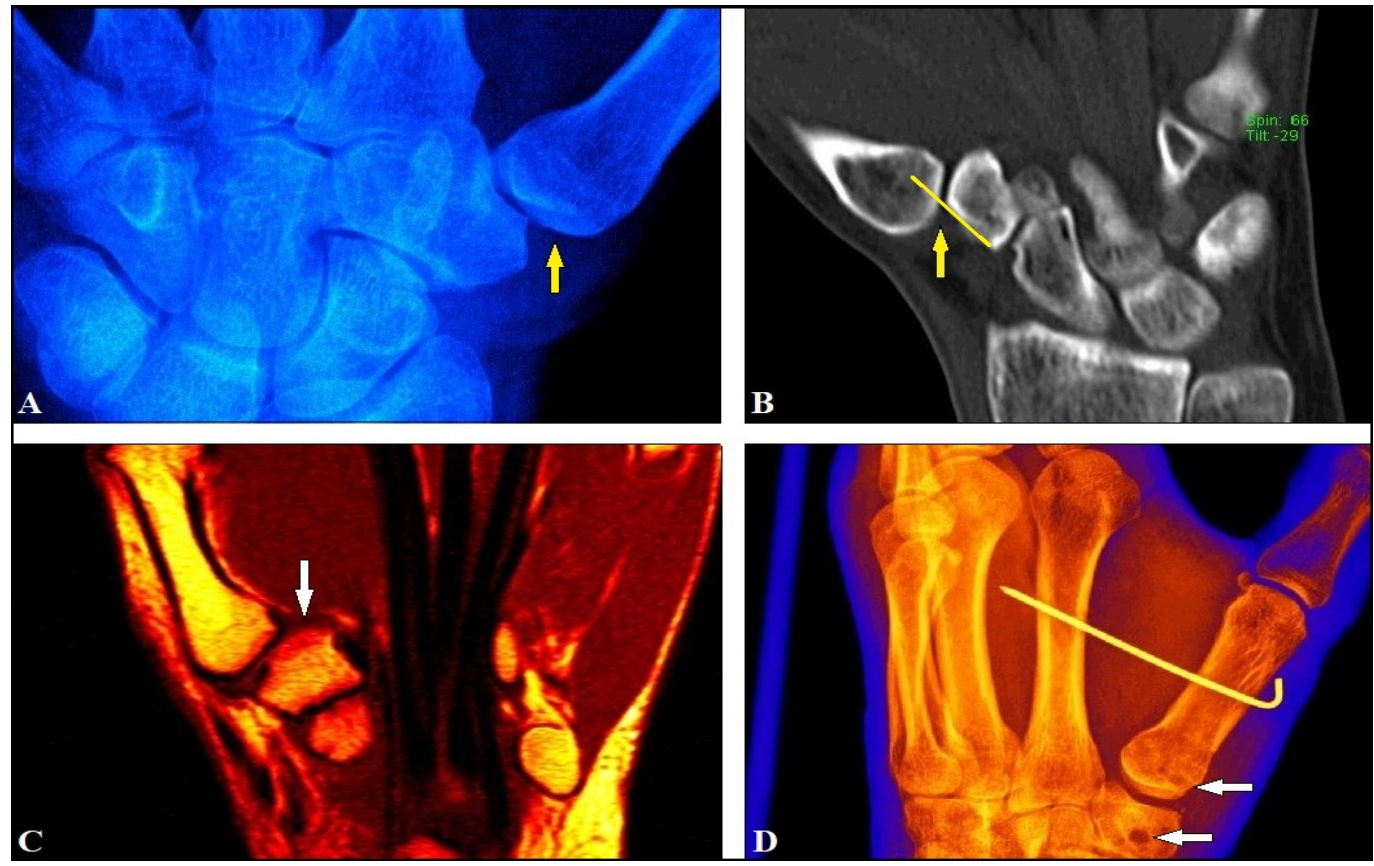

Figure 12 (Case Presentation 5): (A) Static PA radiograph showing marked radial subluxation at the CMCJ I (yellow arrow). (B) CT scan demonstrating subluxation at CMCJ I with more than $50 \%$ of full width of the base of the 1st metacarpal bone (yellow arrow and line). (C) MRI showing disruption of the intermetacarpal ligament (white arrow). (D) Postoperative radiograph demonstrating a correct aligned CMCJ I, metacarpal transfixation was done with $1 \mathrm{~K}$-wire only, note the transverse bony tunnels through the trapez bone and the base of the 1st metacarpal bone (white arrows)

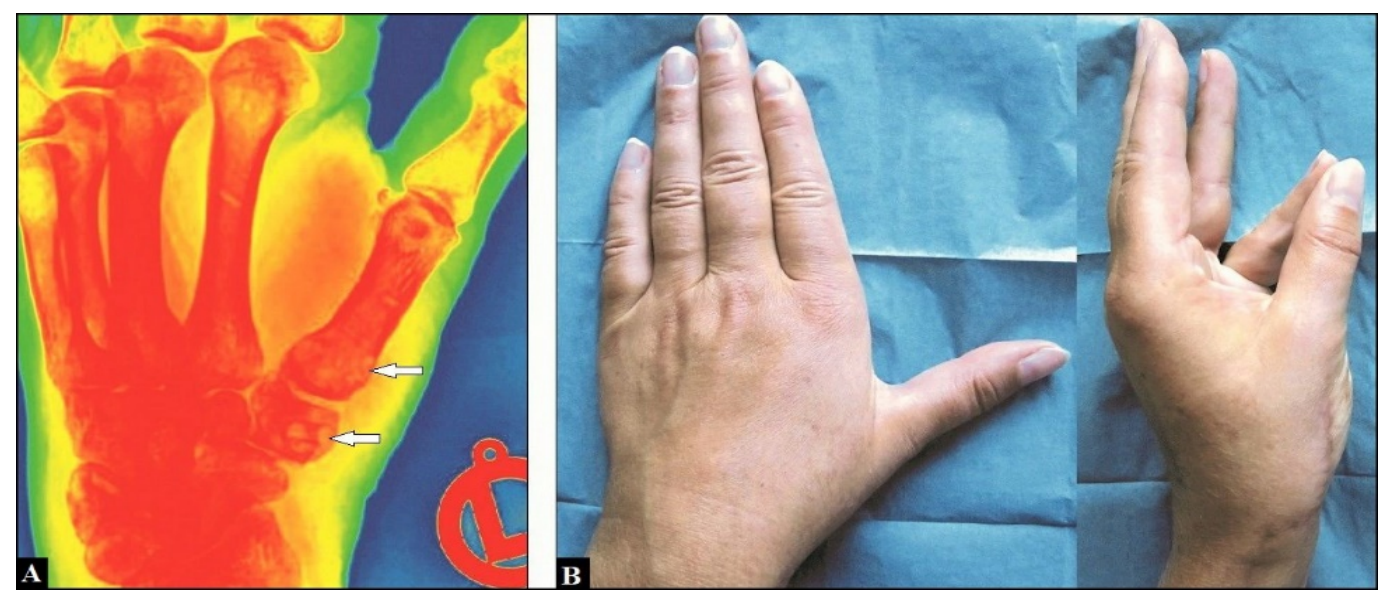

Figure 13 (Case Presentation 5, 6-months follow-up): (A) PA radiograph demonstrating unchanged correct CMCJ I alignment, note the transverse bony tunnels through the trapez bone and the base of the 1st metacarpal bone (white arrows). (B) Clinical photographs showing excellent stable restoration of thumb's function

\section{Declarations}

The author declares that he has none conflict of interests concerning this article.

\section{Acknowledgements}

None.

\section{References}

1. Dobyns JH, Linscheid RL, Cooney WP 3rd (1983) Fractures and dislocations of the wrist and hand, then and now. J Hand Surg Am 8: 687-690. [Crossref]

2. Ebelin M, Soubeyrand M, Idrissi R (2010) Luxaciones carpometacarpianas. Encyclopédie médico chirurgicale - Aparato Locomotor 43: 1-11.

3. Cates RA, Rhee PC, Kakar S (2016) Multiple Volar Carpometacarpal Dislocations: Case Report/Review of the Literature. J Wrist Surg 5: 236-240. [Crossref]
4. Lefere M, Dallaudière B, Omoumi P, Cyteval C, Larbi A (2016) Rare carpometacarpal dislocations. Orthop Traumatol Surg Res 102: 813-816. [Crossref]

5. Hartwig RH, Louis DS (1979) Multiple carpometacarpal dislocations. A review of four cases. J Bone Joint Surg Am 61: 906-908. [Crossref]

6. Bajekal RA, Kotwal PP, Menon D (1992) Closed volar dislocation of the four ulnar carpometacarpal joints. Injury 23: 355-356. [Crossref]

7. Fisher M, Rogers L, Hendrix R (1983) Systematic approach to identifying fourth and fifth carpometacarpal joint dislocations. AJR Am J Roentgenol 140: 319-324. [Crossref]

8. Fisher M, Rogers L, Hendix R (1983) A systematic approach to the diagnosis of carpometacarpal dislocations. Radiographics 2: 612-627.

9. Reinsmith LE, Garcia-Elias M, Gilula LA (2013) Traumatic axial dislocation injuries of the wrist. Radiology 267: 680-689. [Crossref]

10. Garcia-Elias M, Bishop AT, Dobyns JH, Cooney WP, Linscheid RL (1990) Transcarpal carpometacarpal dislocations, excluding the thumb. J Hand Surg Am 15: 531-540. [Crossref] 
11. Gangloff D, Mansat P, Gaston A, Apredoaei C, Rongières M (2007) Carpometacarpal dislocation of the fifth finger: descriptive study of 31 cases. Chir Main 26: 206-213. [Crossref]

12. Diez E, Marti D, Aramburo F, Mendez JM (1997) Multiple carpometacarpal dislocations. Five Cases. Ann Chir Main Memb Super 16: 300-304. [Crossref]

13. KleinmanWB, Grantham SA (1978) Multiple volar carpometacarpal joint dislocation. Case report of traumatic volar dislocation of the medial four carpometacarpal joint in a child and review of the literature. J Hand Surg Am 3: 377-382. [Crossref]

14. Pundkare GT, Patil AM (2015) Carpometacarpal Joint Fracture Dislocation of Second to Fifth Finger. Clin Orthop Surg 7: 430-435. [Crossref]

15. Jumeau H, Lechien P, Dupriez F (2016) Conservative Treatment of Carpometacarpal Dislocation of the Three Last Fingers. Case Rep Emerg Med 2016: 4962021. [Crossref]

16. Ardente PDF, Biayna JC, Sarrias JS, Muñoz AN, Coll GF, et al. (2017) Volar Dislocation of Second, Third and Fourth Carpometacarpal Joints in Association with a Bennet's Fracture of the Thumb Carpo-Metacarpal Dislocation: A Case Report. Open Orthop J 11: 1045-1040. [Crossref]

17. Weiland AJ, Lister GD, Villarreal-Rios A (1976) Volar fracture dislocations of the second and third carpometacarpal joints associated with acute carpal tunnel syndrome. J Trauma 16: 672-675. [Crossref]

18. Gore DR (1971) Carpometacarpal dislocation producing compression of the deep branch of the ulnar nerve. J Bone Joint Surg Am 53: 1387-1390. [Crossref]

19. Lin JD, Karl JW, Strauch RJ (2014) Trapeziometacarpal joint stability: the evolving importance of the dorsal ligaments. Clin Orthop Relat Res 472: 1138-1145. [Crossref]

20. Goubier JN, Devun L, Mitton D, Lavaste F, Papadogeorgou E (2009) Normal range-ofmotion of trapeziometacarpal joint. Chir Main 28: 297-300. [Crossref]

21. Goto A, Leng S, Sugamoto K, Cooney WP 3rd, Kakar S, et al. (2014) In vivo pilot study evaluating the thumb carpometacarpal joint during circumduction. Clin Orthop Relat Res 472: 1106-1113. [Crossref]

22. Ladd AL, Weiss AP, Crisco JJ, Hagert E, Wolf JM, et al. (2013) The thumb carpometacarpal joint: anatomy, hormones, and biomechanics. Instr Course Lect 62: 165-179. [Crossref]

23. Turker T, Thirkannad S (2011) Trapezio-metacarpal arthritis: The price of an opposable thumb! Indian J Plast Surg 44: 308-316. [Crossref]

24. Valentino M, Rapisarda V (2002) [Rhizarthrosis of the thumb in ironing workers]. Med Lav 93: 80-86. [Crossref]

25. Fontana L, Neel S, Claise JM, Ughetto S, Catilina P (2007) Osteoarthritis of the thumb carpometacarpal joint in women and occupational risk factors: a case-control study. $J$ Hand Surg Am 32: 459-465. [Crossref]

26. Glickel S, Barron A, Catalano L (2005) Dislocations and ligament injuries in the digits. In: Green D, Hotchkiss R, Pederson W, Wolfe S. Green's operative hand surgery. 5th ed. Philadelphia,Pennstlvania: Churchill livingstone; 382-386.

27. Kural C, Malkoc M, Ugras AA, Sen A (2002) Isolated carpometacarpal dislocation of the thumb: a case report. Acta Orthop Traumatol Turc 36: 446-448. [Crossref]

28. Schmidt I (2015) Surgical Treatment Options in Thumb Carpometacarpal Osteoarthritis: A Recent Literature Overview Searching for Practice Pattern with Special Focus on Total Joint Replacement. Curr Rheumatol Rev 11: 39-46. [Crossref]
29. Schmidt I (2018) Treatment Options for Thumb Carpometacarpal Joint Osteoarthritis with an Update to the Arpe ${ }^{\mathrm{TM}}$ Prosthesis. Recent Adv Arthroplast 2: 55-63.

30. Hove LM (1993) Fractures of the hand. Distribution and relative incidence. Scand $J$ Plast Reconstr Surg Hand Surg 27: 317-319. [Crossref]

31. Bosmans B, Verhofstad MH, Gosens T (2008) Traumatic thumb carpometacarpal joint dislocations. J Hand Surg Am 33: 438-441. [Crossref]

32. Simonian PT, Trumble TE (1996) Traumatic dislocation of the thumb carpometacarpal joint: early ligamentous reconstruction versus closed reduction and pinning. $J$ Hand Surg Am 21: 802-806. [Crossref]

33. Strauch RJ, Behrman MJ, Rosenwasser MP (1994) Acute dislocation of the carpometacarpal joint of the thumb: an anatomic and cadaver study. J Hand Surg Am 19: 93-98. [Crossref]

34. Annappa R, Kotian P, P JA, Mudiganty S (2015) Ligamentous Reconstruction of Traumatic Dislocation of Thumb Carpometacarpal Joint: Case Report and Review of Literature. J Orthop Case Rep 5: 79-81. [Crossref]

35. Watt N, Hooper G (1987) Dislocation of the trapezio-metacarpal joint. J Hand Surg Br 12: 242-245. [Crossref]

36. Sawalha (2008) Volar dislocation of the thumb carpometacarpal joint: A case report Injury extra 39: 332-334.

37. Farzan M, Siassi M, Espandar R (2002) Thumb carpometacarpal joint volar dislocation: a case report. Acta Med Iran 40: 52-54.

38. Shah J, Patel M (1983) Dislocation of the carpometacarpal joint of the thumb. A report of four cases. Clin Orthop Relat Res : 175: 166-269. [Crossref]

39. Fotiadis E, Svarnas T, Lyrtzis C, Papadopoulos A, Akritopoulos P, et al. (2010) Isolated thumb carpometacarpal joint dislocation: a case report and review of the literature. $J$ Orthop Surg Res 5:16. [Crossref]

40. Imaeda T, An KN, Cooney WP 3rd, Linscheid R (1993) Anatomy of trapeziometacarpa ligaments. J Hand Surg Am 18: 226-231. [Crossref]

41. Lahiji F, Zandi R, Maleki A (2015) First Carpometacarpal Joint Dislocation and Review of Literatures. Arch Bone Jt Surg 3: 300-303. [Crossref]

42. Rust PA, Ek ETH, Tham SKY (2017) Assessment of normal trapeziometacarpal joint alignment. J Hand Surg Eur Vol 42: 605-609. [Crossref]

43. Hirschmann A, Sutter R, Schweizer A, Pfirrmann CW (2013) The carpometacarpal joint oft he thumb: MR appearance in asymptomatic volunteers. Skeletal Radiol 42 1105-1112. [Crossref]

44. Jacobsen CW, Elberg JJ (1988) Isolated carpometacarpal dislocation of the thumb Scand J Plast Reconstr Surg 22: 185-186.

45. Akmaz I, Kiral A, Pehlivan O, Solakoglu C (2003) Ligament reconstruction fo the chronic instability of the traumatic thumb carpometacarpal joint. Acta Orthop Traumatol Turc 37: 237-243. [Crossref]

46. Jeong C, Kim HM, Lee SU, Park IJ (2012) Bilateral carpometacarpal joint dislocations of the thumb. Clin Orthop Surg 4: 246-248. [Crossref]

47. Iyengar K, Gandham S, Nadkarni J, Loh W (2013) Modified Eaton-Littler's Reconstruction for Traumatic Dislocation of the Carpometacarpal Joint of the Thumb-A Case Report and Review of Literature. J Hand Microsurg 5: 36-42. [Crossref]

Copyright: (C2018 Schmidt I. This is an open-access article distributed under the terms of the Creative Commons Attribution License, which permits unrestricted use, distribution, and reproduction in any medium, provided the original author and source are credited. 